\title{
DESEMPENHO DE FUNÇÕES DE DENSIDADE PROBABILÍSTICAS PARA DESCREVER A DISTRIBUIÇÃO DIAMÉTRICA DE Pinus taeda, NA REGIÃO DE CAÇADOR, SC
}

\author{
Saulo Jorge Téo ${ }^{1}$, Júlio César Bianchi ${ }^{2}$, Adriano Peloso ${ }^{2}$, Paulo Roberto $\mathrm{Nava}^{2}$, Alan Marcon ${ }^{2}$, \\ Tiago Ehlers ${ }^{2}$, Reinaldo Hoinacki da Costa ${ }^{3}$ \\ ${ }^{1}$ Eng. Florestal, M.Sc., UNOESC, Xanxerê, SC, Brasil - sauloteo@yahoo.com.br \\ ${ }^{2}$ Acadêmico de Engenharia Florestal, UNOESC, Xanxerê, SC, Brasil - julio_xxe@ hotmail.com; \\ adrianopelosobel@yahoo.com.br;paulorobertonava@hotmail.com;marcon.alan@gmail.com; ehlers6@gmail.com \\ ${ }^{3}$ Eng. Florestal, Juliana Florestal Ltda., Caçador, SC, Brasil - reinaldo@ frameport.com.br \\ Recebido para publicação: 22/02/2012 - Aceito para publicação: 18/12/2012
}

\begin{abstract}
Resumo
O objetivo deste trabalho foi analisar as funções de densidade probabilísticas (FDP) Normal, LnNormal, Sb de Johnson, Weibull 3P, Gamma, Beta e Weber, para descrever as mudanças na estrutura diamétrica de povoamentos de Pinus taeda L., na região de Caçador (SC), em diferentes idades e classes de sítio. O processamento dos dados foi realizado por meio da ferramenta Solver, do software MS Excel 2010, a qual utiliza o algoritmo linear de gradiente reduzido generalizado (GRG) na interação dos parâmetros. Verificou-se que a FDP Sb de Johnson e Weibull 3 Papresentaram os melhores desempenhos. Para a avaliação da aderência das FDP, é recomendada a utilização das estatísticas $R^{2}, R_{a j .}^{2}$, syx e syx\%, além do teste de Kolmogorov-Smirnov, em todos os casos, especialmente quando houver número de observações superior a 5.000. Geralmente, houve um aumento da amplitude dos valores dos diâmetros e um achatamento da distribuição diamétrica com o avanço da idade e com a melhora da produtividade do sítio. Com o progresso da idade, há um aumento dos valores do diâmetro máximo e do diâmetro modal das distribuições, para as classes de sítio de maior produtividade, porém o mesmo não ocorre para o sítio menos produtivo.

Palavras-chave: Estrutura horizontal; teste de Kolmogorov-Smirnov; função de densidade probabilística; sítio florestal.
\end{abstract}

\begin{abstract}
Performance of probability density functions in order to describe diameter distribution of Pinus taeda, in the region of Caçador, SC. This research aims to analyze probability of density functions (pdf) Normal, LnNormal, Johnson Sb, 3PWeibull, Gamma, Beta and Weber in order to describe diameter changes in Pinus taeda L. plantations structure, in the region of Caçador - SC, Brazil, at different age and site classes. The data processing was carried out by Solver tool of the software MSExcel2010, using the linear algorithm of generalized reduced gradient (GRG) for interaction of parameters. As result, Johnson Sb and 3PWeibull presented the best performances. For the pdf adherence evaluation, it was recommended the employment of $R^{2}, R_{a d}^{2}$, syx e syx \%statistics, besides the Kolmogorov-Smirnov test, in any situation, specially, when there is more than 5,000 observations. Generally, there was an increasing in the range of diameter values and a flatness of diameter distribution at advancing age and improvement of the site productivity. At age advancing, there was an increasing of maximum diameter and modal diameter values of distributions, for the higher productivity site classes, on the other hand, the same did not occur for the low productivity site class.

Keywords: Horizontal structure; Kolmogorov-Smirnov test; probability density function; forestry site.
\end{abstract}

\section{INTRODUÇÃO}

A palavra estrutura significa a distribuição, disposição ou organização das partes de um todo. Em se tratando de uma floresta, a estrutura diz respeito às árvores e como elas se distribuem no espaço e nas diferentes classes de tamanho. Dessa forma, a distribuição diamétrica trata da disposição das árvores com relação às posições de maior ou menor diâmetro em uma floresta, caracterizando-se pelo agrupamento das 
árvores em classes de diâmetro à altura do peito (d), a qual, conforme Loetschet al. (1973), podem ser representadas na forma de tabela, histograma de frequências ou de um modelo, nesse último caso, as Funções de Densidade Probabilísticas (FDP).

De acordo com Loetsch et al. (1973), existem algumas razões pelas quais o diâmetro ou a circunferência à altura do peito seja de particular importância, assim como a distribuição diamétrica de uma floresta. O diâmetro é uma variável de fácil acesso e medição. É uma medida básica da qual derivam outras medidas e cálculos. O diâmetro é importante para medições de densidade da floresta e a distribuição diamétrica serve de base para estudos de sortimento florestal, melhor aproveitamento da madeira e planejamento da produção florestal.

Arce (2004) e Bartoszeck et al.(2004) acrescentaram que a distribuição diamétrica é a ferramenta mais simples e poderosa para caracterizar a estrutura de uma floresta, e a variável diâmetro, obtida por medição direta das árvores, é bem correlacionada com outras variáveis importantes, tais como volume, qualidade da madeira e custos de exploração.

De acordo com Clutter et al. (1983), modelos implícitos de crescimento e produção também são chamados de modelos de distribuição diamétrica, pois empregam classes diamétricas como unidade básica da modelagem. Os modelos de distribuição diamétrica têm sido bastante utilizados, tanto em plantios florestais como em florestas nativas, pois fornecem informações detalhadas quanto à estrutura das florestas.

Embora o agrupamento das árvores de uma floresta em classes diamétricas elimine muitos detalhes originais, esse procedimento traz a vantagem de visualização do aspecto geral da floresta e de sua composição quanto à grandeza e amplitude da variável diâmetro à altura do peito. A distribuição diamétrica caracteriza o estoque em crescimento de uma floresta e permite a diferenciação entre tipologias florestais, a identificação da amplitude de variação e média dos diâmetros e a identificação da intensidade de regeneração natural da floresta.

Dentre os tipos de distribuição diamétrica de florestas, podem-se citar a distribuição unimodal, a distribuição decrescente e a distribuição multimodal (LOETSCH et al., 1973; PRODAN et al., 1997). A distribuição unimodal caracteriza-se por apresentar um único valor para a moda e é característica de povoamentos florestais equiâneos. A distribuição decrescente, também chamada de J-invertido, é caracterizada pela diminuição do número de árvores à medida que o diâmetro aumenta e é característica de florestas naturais onde ocorre regeneração contínua, constituída de várias espécies em várias classes de idade. Por fim, a distribuição multimodal apresenta mais de um ponto de maior frequência, entretanto é raramente encontrada na natureza.

A evolução das curvas de distribuição unimodal é descrita pelos parâmetros de assimetria e curtose. A assimetria ocorre quando a média, mediana e moda da distribuição não coincidem, ou seja, há um afastamento da simetria da distribuição de frequência com relação à distribuição Normal. Já a curtose é definida como o grau de achatamento de uma distribuição em relação à curva Normal (LOETSCH et al., 1973; MACHADO et al., 2006).

A representação da distribuição diamétrica por meio de funções de densidade probabilística, com baixos níveis de erro de estimativa, possui um grande valor para empresas e profissionais que trabalham no planejamento e manejo florestal, tendo em vista que, quanto mais se conhece sobre a floresta, melhor podem-se planejar as atividades de industrialização e beneficiamento da madeira, bem como otimizar os investimentos e a lucratividade dos povoamentos florestais.

Diante disso, a hipótese da presente pesquisa é que funções de densidade probabilísticas podem representar com precisão a distribuição diamétrica de povoamentos de Pinus taeda, bem como descrever mudanças ocorridas na distribuição diamétrica dessa espécie com a evolução da sua idade e nas diferentes classes de sítio florestal.

O objetivo deste estudo foi determinar qual função de densidade probabilística melhor descreve a distribuição diamétrica em povoamentos de Pinus taeda na região de Caçador (SC), em diferentes idades e classes de sítio, bem como analisar as mudanças das distribuições de diâmetro sob as diferentes idades e classes de sítio.

\section{MATERIAL E MÉTODOS}

Os dados para a realização desta pesquisa foram obtidos em áreas da empresa Juliana Florestal Ltda., localizadas na região de Caçador (SC). Além de Caçador, os povoamentos de Pinus taeda 
utilizados na execução deste estudo são provenientes dos municípios de Macieira, Calmon, Timbó Grande, Lebon Régis, Rio das Antas e Santa Cecília.

Segundo Prates et al. (1989), o município de Caçador apresenta o clima Cfb (clima subtropical com verões brandos) da classificação de Köppen. A temperatura média anual desse município varia de 14 a $16^{\circ} \mathrm{C}$. A precipitação pluviométrica total anual é de 1300 a $2000 \mathrm{~mm}$ e a umidade relativa do ar média é de 78,1 a 82,9\%. Quanto à vegetação, originalmente ocorriam a Floresta Ombrófila Mista e Campos do Planalto. As classes predominantes de solos da região dos povoamentos em estudo são, predominantemente, Latossolos e Cambissolos.

Os dados para a realização deste estudo foram obtidos por meio de medições de parcelas permanentes e temporárias em povoamentos de Pinus taeda, realizadas de 2007 a 2010. Anualmente, foram realizadas medições em parcelas de área fixa, variando de 400 a $800 \mathrm{~m}^{2}$ e distribuídas de maneira aleatória nos povoamentos de Pinus taeda da região de estudo.

Os povoamentos apresentavam idades de 5 a 8,9 anos, de acordo com suas respectivas datas de plantio e de medição das parcelas do inventário florestal. Para o processamento dos dados, as parcelas foram agrupadas em classes de idade, como segue: de 5 a 5,9 anos, de 6 a 6,9 anos, de 7 a 7,9 anos e de 8 a 8,9 anos. Após esse agrupamento, foi realizada a classificação de sítios de acordo com Téo et al. (2011a), em que foram consideradas as classes de sítio I, II e III, cujos índices de sítio na idade de referência de 24 anos são 35,91, 30,14 e 24,36 m, respectivamente (Tabela 1).

Tabela 1. Número total de árvores de Pinus taeda medidas por classe de idade e classe de sítio.

Table 1. Total number of Pinus taeda trees measured by age and site class.

\begin{tabular}{lcccc}
\hline \multirow{2}{*}{ Idade (anos) } & \multirow{2}{*}{ Número de árvores } & \multicolumn{3}{c}{ Classes de Sítio } \\
\cline { 3 - 5 } & 22.046 & I & II & III \\
\hline $5-5,9$ & 52.886 & 4.883 & 16.452 & 4.724 \\
$6-6,9$ & 35.734 & 1.182 & 43.045 & 4.958 \\
$7-7,9$ & 17.051 & 1.317 & 31.148 & 3.404 \\
$8-8,9$ & 127.717 & 8.252 & 15.383 & 351 \\
\hline Total & & & & 106.028 \\
\hline
\end{tabular}

O processamento dos dados se constituiu no agrupamento dos dados de diâmetro em diferentes classes diamétricas com intervalos de $3 \mathrm{~cm}$, na classificação de sítios, no ajuste das funções de densidade probabilística Normal, Ln-Normal, Beta, Gamma, Weibull 3P, Sb de Johnson e Weber, na realização do teste de aderência de Kolmogorov-Smirnov, no cálculo das estatísticas de ajuste e precisão $R^{2}, R_{a j}^{2}$, syx e syx\% e no cálculo dos parâmetros de assimetria e curtose.

A tabela 2 apresenta as funções de densidade probabilísticas testadas, com suas condicionantes, para distribuição diamétrica de Pinus taeda em diferentes idades e classes de sítio, na região de Caçador (SC).

Para realizar o ajuste das FDP, foi criada uma planilha no software MS Excel 2010, onde se obtiveram as frequências por classe diamétrica e os valores iniciais dos parâmetros de cada função. Esses valores iniciais, juntamente com as frequências por classe diamétrica, foram então processados no software MS Excel, por meio da ferramenta Solver, a qual utilizou o algoritmo de gradiente reduzido generalizado (GRG) na interação dos parâmetros das funções, com objetivo de minimizar a soma dos quadrados dos resíduos.

Os critérios utilizados para a seleção da função de densidade probabilística de melhor aderência foram as estatísticas de ajuste e precisão $R^{2}, R_{a j}^{2}$, syx esyx\%, calculadas com os valores observados e estimados pelas FDP do número de árvores por classe diamétrica. Tais estatísticas de ajuste e precisão são amplamente conhecidas no meio florestal e sua descrição detalhada pode ser encontrada em Draper e Smith (1998) e Montgomery et al. (2006).

Além das estatísticas de ajuste e precisão, as funções de densidade probabilísticas foram submetidas ao teste de Kolmogorov-Smirnov, o qual compara a frequência acumulada observada e a estimada pela FDP em seu ponto de maior diferença. Neste estudo, o valor da maior diferença entre a frequência observada e a estimada $\left(D_{\text {calc }}\right)$ foi comparado com um valor tabelado $\left(D^{\prime}\right)$ para $1 \%$ de nível de significância do teste bilateral. 
Tabela 2. Funções de densidade probabilísticas testadas para descrever a distribuição diamétrica de povoamentos de Pinus taeda em diferentes classes de idade e classes de sítio.

Table 2. Probability density functions tested for describing the diameter distribution of Pinus taeda stands at different age and site classes.

\begin{tabular}{|c|c|c|}
\hline Nome & Funções de densidade probabilísticas (FDP) & $\begin{array}{c}\text { Fatores } \\
\text { condicionantes }\end{array}$ \\
\hline Normal & $f x=\frac{1}{\sigma * \overline{2 \pi}} * e^{-\frac{1}{2} * \frac{(x-\mu)^{2}}{\sigma^{2}}}$ & $\begin{aligned} \sigma & >0 \\
-\infty & <x<+\infty \\
-\infty & <\mu<+\infty\end{aligned}$ \\
\hline $\begin{array}{l}\text { Ln- } \\
\text { Normal }\end{array}$ & $f x=\frac{1}{\sigma * \overline{2 \pi}} * e^{-\frac{1}{2} * \frac{(\ln x-\mu)^{2}}{\sigma^{2}}}$ & $\begin{aligned} x & \geq x_{\min } \\
\sigma & >0 \\
-\infty & <x<+\infty \\
-\infty & <\mu<+\infty\end{aligned}$ \\
\hline $\begin{array}{l}\text { Weibull } \\
3 \mathrm{P}\end{array}$ & $f x=\frac{c}{b} * \frac{x-a}{b}^{(c-1)} * e^{-\frac{x-a}{b}^{c}}$ & $\begin{array}{c}x \geq x_{\min } \\
-\infty<x_{\min } \\
<+\infty \\
b, c>0 \\
a \geq 0\end{array}$ \\
\hline $\begin{array}{l}\text { Sb de } \\
\text { Johnson }\end{array}$ & $f x=\frac{\delta}{\overline{2 \pi}} * \frac{\lambda}{x-\varepsilon \lambda+\varepsilon-x} * e^{-\frac{1}{2} \gamma+\delta * \ln \frac{x-\varepsilon}{\lambda+\varepsilon-x}}$ & $\begin{aligned} \varepsilon & <x<+\lambda \\
-\infty & <\gamma<+\infty \\
\lambda & >0 \\
\delta & >0\end{aligned}$ \\
\hline Gamma & $f x=\frac{x-x_{\min }^{\alpha-1} * e^{-\frac{x-x_{\min }}{\beta}}}{\beta^{\alpha} * \Gamma \alpha}$ & $\begin{array}{l}x \geq x_{\min } \\
\alpha, \beta>0 \\
-\infty<x_{\min } \\
<+\infty\end{array}$ \\
\hline Beta & $f x=\frac{\Gamma \alpha+\beta}{\Gamma \alpha \Gamma \beta} * \frac{1}{x_{\text {máx }}-x_{\text {mín }}^{\alpha+\beta-1}} * x-x_{\min }^{\alpha-1} * x_{m a ́ x}-x^{\beta-1}$ & $\begin{array}{c}x_{\min }<x<x_{\text {máx }} \\
\alpha, \beta>0 \\
-\infty<x_{\min } \\
<x_{\operatorname{má}} \\
x_{\min }<x_{\operatorname{má}} \\
<+\infty\end{array}$ \\
\hline Weber & $f x=\frac{x^{a}}{b+c * x^{d}}$ & $\begin{array}{c}0 \leq x<+\infty \\
d \geq a+1 \\
d \neq a+2 \\
d \neq a+3 \\
a, b, c, d>0\end{array}$ \\
\hline
\end{tabular}

$f x$ : função de densidade probabilística da variável $x ; x$ : variável aleatória; $\mu$ : média dos valores de $x ; \sigma$ :desvio padrão de $x$; $x_{\min }=$ valor mínimo de $x ; x_{m a ́ x}:$ valor máximo de $x ; \pi: 3,14159 \ldots ; e$ : constante de Euler $(2,718281829 \ldots) ; a, b, c, d, \alpha, \beta, \gamma, \delta, \varepsilon, \lambda$ : parâmetros a serem estimados.

Para a seleção das melhores funções de densidade probabilísticas, foi realizada uma classificação com base em cinco critérios de seleção: $R^{2}, R_{a j}^{2}$, syx, syx $\%$ e $D_{c a l c}$ do teste de aderência de Kolmogorov-Smirnov. A qualidade da aderência de cada função foi classificada de acordo com um ranking formado por todos os critérios anteriormente citados. Foram atribuídas pontuações tanto menores quanto melhores os critérios calculados. Ao final da pontuação, a função que apresentou a menor soma foi selecionada como a função de densidade probabilística de melhor aderência.

Para descrever as formas e a evolução das curvas de distribuição, procedeu-se ao cálculo dos parâmetros de assimetria $\left(\gamma_{1}\right)$ e curtose $\left(\gamma_{2}\right)$. O parâmetro de assimetria $\left(\gamma_{1}\right)$ foi obtido por meio do quociente entre o terceiro momento centrado na média $\left(m_{3}\right)$ e o cubo do desvio padrão. O parâmetro de curtose $\left(\gamma_{2}\right)$ é definido pelo quociente entre o quarto momento centrado na média $\left(m_{4}\right)$ e o quadrado da variância, menos três.

\section{RESULTADOS E DISCUSSÃO}

A tabela 3 apresenta a distribuição diamétrica observada para os povoamentos de Pinus taeda da região de Caçador (SC), de acordo com a idade e a classe de sítio, as quais foram necessárias para o ajuste das funções de densidade probabilísticas. 
Tabela 3. Distribuição diamétrica observada para os povoamentos de Pinus taeda, com idades entre 5 e 8,9 anos, para classes de sítio I, II e III.

Table 3. Observed diameter distribution for Pinus taeda stands, with ages ranging from 5 to 8.9 years, and site classes I, II and III.

\begin{tabular}{|c|c|c|c|c|c|c|c|c|c|c|c|c|}
\hline \multirow{3}{*}{$\begin{array}{l}\text { Classe } \\
\text { diamétrica } \\
(\mathrm{cm})\end{array}$} & \multicolumn{12}{|c|}{ Número de árvores por hectare (árv./ha) } \\
\hline & \multicolumn{3}{|c|}{$5-5,9$ anos } & \multicolumn{3}{|c|}{$6-6,9$ anos } & \multicolumn{3}{|c|}{$7-7,9$ anos } & \multicolumn{3}{|c|}{$8-8,9$ anos } \\
\hline & I & II & III & $\mathbf{I}$ & II & III & $\mathbf{I}$ & II & III & $\mathbf{I}$ & II & III \\
\hline $3-6$ & 4 & 6 & 10 & 3 & 3 & 1 & 7 & 3 & 0 & 10 & 6 & 4 \\
\hline $6-9$ & 25 & 42 & 74 & 16 & 23 & 23 & 15 & 19 & 12 & 22 & 23 & 17 \\
\hline $9-12$ & 92 & 201 & 348 & 41 & 80 & 103 & 50 & 52 & 51 & 54 & 46 & 91 \\
\hline $12-15$ & 424 & 734 & 878 & 203 & 376 & 482 & 202 & 211 & 223 & 123 & 160 & 339 \\
\hline $15-18$ & 662 & 504 & 274 & 477 & 645 & 735 & 457 & 486 & 563 & 321 & 368 & 677 \\
\hline $18-21$ & 295 & 76 & 10 & 524 & 364 & 227 & 501 & 539 & 547 & 404 & 508 & 289 \\
\hline $21-24$ & 34 & 2 & - & 224 & 60 & 7 & 284 & 229 & 134 & 432 & 404 & 33 \\
\hline $24-27$ & 2 & - & - & 21 & 2 & - & 66 & 25 & 5 & 209 & 100 & - \\
\hline $27-30$ & - & - & - & 1 & - & - & 9 & 2 & - & 48 & 12 & - \\
\hline $30-33$ & - & - & - & - & - & - & 1 & - & - & 6 & 1 & - \\
\hline $33-36$ & - & - & - & - & - & - & - & - & - & 1 & - & - \\
\hline Total & 1.536 & 1.566 & 1.595 & 1.512 & 1.551 & 1.579 & 1.593 & 1.567 & 1.536 & 1.631 & 1.628 & 1.450 \\
\hline
\end{tabular}

Por meio da tabela 3, é possível identificar o efeito do sítio no desenvolvimento do diâmetro, pois o número de classes diamétricas, assim como o diâmetro máximo, diminui à medida que o sítio piora. Também se pode observar, na tabela 3, que o diâmetro das classes com maior frequência possui uma tendência de redução com a diminuição da qualidade de sítio.

Com relação ao efeito da idade sobre a distribuição diamétrica, pode-se perceber que ocorre maior número de classes diamétricas com o avanço da idade, o que demonstra uma maior amplitude na distribuição dos diâmetros dentro das classes diamétricas. Carelli Netto (2008), Leite et al. (2005), Machado et al. (2006), Nogueira et al. (2006) e Téo et al. (2011b), no estudo da dinâmica da distribuição diamétrica de diversas espécies no Brasil, também encontraram uma maior amplitude dos diâmetros com o avanço da idade. E, naturalmente, também é possível observar na tabela 3 o aumento do diâmetro da classe de maior frequência com o avanço da idade.

Nas tabelas 4, 5, 6 e 7 pode-se observar a aderência das funções de densidade probabilísticas para povoamentos de Pinus taeda na região de Caçador (SC), com idades de 5 a 8,9 anos, para as classes de sítio I, II e III.

Na tabela 4, que apresenta a aderência das FDP para a classe de idade de 5 a 5,9 anos, observa-se que a função Normal apresentou o melhor desempenho para as classes de sítio I e II, enquanto que a função Weibull apresentou o melhor desempenho para a classe de sítio III. Ainda entre as funções de melhor aderência para as idades de 5 a 5,9 anos, está a função $\mathrm{Sb}$ de Johnson. Por outro lado, as funções de Weber, Ln-Normal e Gamma apresentaram os piores resultados de aderência para essa classe de idade.

$\mathrm{Na}$ tabela 5, a qual apresenta o desempenho das FDP para a classe de idade de 6 a 6,9 anos, pode-se observar que a função $\mathrm{Sb}$ de Johnson apresentou melhor aderência para as classes de sítio I e II e a função Weibull para a classe de sítio III. A função Beta, Normal e Sb de Johnson também apresentaram bons desempenhos para as classes de sítio I, II e III, respectivamente. Entre as funções que não apresentaram bons resultados de aderência, podem-se citar função de Weber, Ln-Normal e Gamma.

A tabela 6 mostra o desempenho das FDP para idades entre 7 e 7,9 anos, com a função $\mathrm{Sb}$ de Johnson apresentando a melhor aderência em todas as classes de sítio, seguida da função Normal na classe de sítio I e da função Weibull nas classes de sítio II e III. A função Weber, Gamma e Ln-Normal apresentaram os piores resultados para as idades de 7 a 7,9 anos.

Na tabela 7, que demonstra a aderência das FDP para as idades de 8 a 8,9 anos, a função Weibull apresentou o melhor desempenho em todas as classes de sítio, seguida da função Sb de Johnson. A função Weber, Gamma e Ln-Normal apresentaram os piores resultados para as idades de 8 a 8,9 anos. 
Tabela 4. Aderência das funções de densidade probabilísticas à distribuição diamétrica de povoamentos de Pinus taeda, com idades de 5 a 5,9 anos, por classe de sítio.

Table 4. Adherence of the probability density functions to diameter distribution of Pinus taeda stands, with ages ranging from 5 to 5.9 years, by site classes.

\begin{tabular}{|c|c|c|c|c|c|c|c|c|}
\hline $\begin{array}{l}\text { Classe } \\
\text { de sítio }\end{array}$ & FDP & Ranking & $R^{2}$ & $R_{a j}^{2}$ & $\begin{array}{c}\text { syx } \\
\text { (árv./ha) }\end{array}$ & $\boldsymbol{s y} \boldsymbol{y} \%$ & $D^{\prime}$ & $D_{\text {calc }}$ \\
\hline \multirow{7}{*}{ I } & Normal & $1^{\mathrm{a}}$ & 0,9986 & 0,9980 & 10,8 & 5,6 & \multirow{7}{*}{0,055} & $-0,021^{\mathrm{ns}}$ \\
\hline & Sb de Johnson & $2^{\mathrm{a}}$ & 0,9983 & 0,9970 & 13,4 & 7,0 & & $-0,018^{\mathrm{ns}}$ \\
\hline & Weibull & $3^{\mathrm{a}}$ & 0,9976 & 0,9967 & 14,1 & 7,3 & & $-0,013^{\mathrm{ns}}$ \\
\hline & Beta & $4^{a}$ & 0,9968 & 0,9926 & 21,1 & 11,0 & & $-0,036^{\mathrm{ns}}$ \\
\hline & Gamma & $5^{\mathrm{a}}$ & 0,9883 & 0,9836 & 31,4 & 16,4 & & $-0,052^{\mathrm{ns}}$ \\
\hline & Ln-Normal & $6^{\mathrm{a}}$ & 0,9871 & 0,9819 & 33,0 & 17,2 & & $-0,058 * *$ \\
\hline & Weber & $7^{\mathrm{a}}$ & 0,9856 & 0,9749 & 38,8 & 20,2 & & $-0,051^{\mathrm{ns}}$ \\
\hline \multirow{7}{*}{ II } & Normal & $1^{\mathrm{a}}$ & 0,9982 & 0,9975 & 13,2 & 7,6 & \multirow{7}{*}{0,013} & $-0,023 * *$ \\
\hline & Sb de Johnson & $2^{\mathrm{a}}$ & 0,9975 & 0,9960 & 16,8 & 9,6 & & $-0,022 * *$ \\
\hline & Beta & $3^{\mathrm{a}}$ & 0,9973 & 0,9947 & 19,5 & 11,2 & & $-0,022 * *$ \\
\hline & Weibull & $4^{a}$ & 0,9955 & 0,9940 & 20,7 & 11,9 & & $-0,031 * *$ \\
\hline & Gamma & $5^{\mathrm{a}}$ & 0,9935 & 0,9913 & 24,9 & 14,3 & & $-0,045 * *$ \\
\hline & Ln-Normal & $6^{\mathrm{a}}$ & 0,9921 & 0,9895 & 27,3 & 15,7 & & $-0,047 * *$ \\
\hline & Weber & $7^{\mathrm{a}}$ & 0,9908 & 0,9853 & 32,3 & 18,6 & & $-0,038 * *$ \\
\hline \multirow{7}{*}{ III } & Weibull & $1^{\mathrm{a}}$ & 0,9998 & 0,9997 & 5,2 & 2,3 & \multirow{7}{*}{0,024} & $-0,009^{\mathrm{ns}}$ \\
\hline & Normal & $2^{a}$ & 0,9947 & 0,9920 & 28,5 & 12,5 & & $-0,041 * *$ \\
\hline & Sb de Johnson & $3^{\mathrm{a}}$ & 0,9933 & 0,9867 & 36,8 & 16,2 & & $-0,039 * *$ \\
\hline & Ln-Normal & $4^{a}$ & 0,9907 & 0,9861 & 37,6 & 16,5 & & $-0,053 * *$ \\
\hline & Gamma & $5^{\mathrm{a}}$ & 0,9903 & 0,9854 & 38,5 & 16,9 & & $-0,051 * *$ \\
\hline & Beta & $6^{\mathrm{a}}$ & 0,9934 & 0,9803 & 44,7 & 19,6 & & $-0,045 * *$ \\
\hline & Weber & $7^{\mathrm{a}}$ & 0,9802 & 0,9603 & 63,5 & 27,9 & & $-0,048 * *$ \\
\hline
\end{tabular}

FDP: função de densidade probabilística; $R^{2}$ : coeficiente de determinação; $R_{a j}^{2}$ : coeficiente de determinação ajustado; syx: erro padrão da estimativa, em árv./ha; syx\%: erro padrão da estimativa em porcentagem; $D^{\prime}$ : valor tabelado do teste de KolmogorovSmirnov $(\alpha: 0,01) ; D_{\text {calc }}$ : valor calculado do teste de Kolmogorov-Smirnov; ${ }^{\text {ns: }}$ não significativo ao nível de $1 \%(\alpha: 0,01)$; **: significativo ao nível de $1 \%(\alpha: 0,01)$; I, II, III :classe de sítio I, classe de sítio II e classe de sítio III, as quais apresentam altura dominante aos 24 anos de idade de 35,91, 30,14 e 24,36 m, respectivamente.

Quando consideradas todas as classes de idade e todas as classes de sítio, a classificação das funções de densidade probabilísticas, quanto ao desempenho para representar a distribuição diamétrica de povoamentos de Pinus taeda na região de Caçador, foi a seguinte: a função Sb de Johnson e a função Weibull ficam com a mesma pontuação, em primeiro lugar, a função Normal ficou com a terceira, a função Beta com a quarta, a função Gamma com a quinta, a função Ln-Normal com a sexta colocação e a função Weber em último lugar, com os ajustes menos precisos.

Bartoszeck et al. (2004) testaram várias FDP para representar a distribuição diamétrica de povoamentos de Mimosa scabrella Bentham, com diferentes idades, índices de sítio e densidades iniciais, na Região Metropolitana de Curitiba (PR). De acordo com os resultados, detectou-se que a função Sb de Johnson foi a de melhor desempenho na maioria dos casos. Segundo Machado et al. (2009a), entre as funções Normal, Log-Normal, Gamma, Beta, Weibull 3P, Weibull 2P e Sb de Johnson para descrever a distribuição diamétrica de Araucaria angustifolia (Bert.) O. Ktze. em Curitiba (PR), a função Normal e $\mathrm{Sb}$ de Johnson apresentaram os melhores desempenhos, considerando o teste de Kolmogorov-Smirnov.

Figura (2010) testou diferentes métodos de ajuste da função Weibull na descrição da estrutura diamétrica de povoamentos de Eucalyptus grandis Hill ex Maiden não desbastados em Telêmaco Borba (PR). De acordo com Figura (2010), há uma melhora na aderência da função Weibull com o aumento da idade dos povoamentos dessa espécie. Ao observar as tabelas 4 a 7 desta pesquisa, é possível notar que os melhores desempenhos da função Weibull ocorreram para as idades mais avançadas, reiterando a tendência encontrada por Figura (2010). Além disso, é possível observar que a função Weibull apresentou sempre o melhor desempenho para a classe de sítio III, exceto para as idades de 7 a 7,9 anos. 
Tabela 5. Aderência das funções de densidade probabilísticas à distribuição diamétrica de povoamentos de Pinus taeda com idades de 6 a 6,9 anos, por classe de sítio.

Table 5. Adherence of the probability density functions to diameter distribution of Pinus taeda stands, with ages ranging from 6 to 6.9 years, by site classes.

\begin{tabular}{|c|c|c|c|c|c|c|c|c|}
\hline $\begin{array}{l}\text { Classe } \\
\text { de sítio }\end{array}$ & FDP & Ranking & $R^{2}$ & $R_{a j}^{2}$ & $\begin{array}{c}s y x \\
\text { (árv./ha) }\end{array}$ & syx\% & $D^{\prime}$ & $D_{\text {calc }}$ \\
\hline \multirow{7}{*}{ I } & Sb de Jonhson & $1^{\mathrm{a}}$ & 0,9995 & 0,9992 & 5,9 & 3,5 & \multirow{7}{*}{0,023} & $-0,009^{\mathrm{ns}}$ \\
\hline & Weibull & $2^{\mathrm{a}}$ & 0,9991 & 0,9987 & 7,3 & 4,4 & & $-0,012^{\mathrm{ns}}$ \\
\hline & Beta & $3^{\mathrm{a}}$ & 0,9984 & 0,9968 & 11,6 & 6,9 & & $-0,021^{\mathrm{ns}}$ \\
\hline & Normal & $4^{\mathrm{a}}$ & 0,9970 & 0,9960 & 13,1 & 7,8 & & $-0,023^{\mathrm{ns}}$ \\
\hline & Gamma & $5^{\mathrm{a}}$ & 0,9797 & 0,9729 & 34,1 & 20,3 & & $-0,061 * *$ \\
\hline & Ln-Normal & $6^{\mathrm{a}}$ & 0,9769 & 0,9692 & 36,3 & 21,6 & & $-0,057 * *$ \\
\hline & Weber & $7^{\mathrm{a}}$ & 0,9785 & 0,9656 & 38,4 & 22,9 & & $-0,053^{* *}$ \\
\hline \multirow{7}{*}{ II } & Sb de Jonhson & $1^{\mathrm{a}}$ & 0,9993 & 0,9990 & 7,2 & 4,7 & \multirow{7}{*}{0,008} & $-0,011 * *$ \\
\hline & Normal & $2^{\mathrm{a}}$ & 0,9990 & 0,9988 & 8,0 & 5,1 & & $-0,017 * *$ \\
\hline & Weibull & $3^{a}$ & 0,9981 & 0,9976 & 11,1 & 7,2 & & $-0,016^{* *}$ \\
\hline & Beta & $4^{\mathrm{a}}$ & 0,9982 & 0,9967 & 13,0 & 8,4 & & $-0,026^{* *}$ \\
\hline & Gamma & $5^{a}$ & 0,9909 & 0,9882 & 24,5 & 15,8 & & $-0,047 * *$ \\
\hline & Ln-Normal & $6^{\mathrm{a}}$ & 0,9891 & 0,9860 & 26,7 & 17,2 & & $-0,056^{* *}$ \\
\hline & Weber & $7^{\mathrm{a}}$ & 0,9891 & 0,9837 & 28,8 & 18,6 & & $-0,054 * *$ \\
\hline \multirow{7}{*}{ III } & Weibull & $1^{\mathrm{a}}$ & 0,9989 & 0,9985 & 10,2 & 5,8 & \multirow{7}{*}{0,023} & $-0,015^{\mathrm{ns}}$ \\
\hline & Normal & $2^{\mathrm{a}}$ & 0,9971 & 0,9961 & 16,5 & 9,4 & & $-0,032 * *$ \\
\hline & Sb de Jonhson & $3^{\mathrm{a}}$ & 0,9969 & 0,9950 & 18,6 & 10,6 & & $-0,028 * *$ \\
\hline & Beta & $4^{\mathrm{a}}$ & 0,9950 & 0,9899 & 26,6 & 15,1 & & $-0,041 * *$ \\
\hline & Ln-Normal & $5^{a}$ & 0,9854 & 0,9806 & 36,9 & 21,0 & & $0,067 * *$ \\
\hline & Gamma & $6^{\mathrm{a}}$ & 0,9792 & 0,9723 & 44,0 & 25,1 & & $0,058 * *$ \\
\hline & Weber & $7^{\mathrm{a}}$ & 0,9745 & 0,9592 & 53,4 & 30,4 & & $0,053 * *$ \\
\hline
\end{tabular}

FDP: função de densidade probabilística; $R^{2}$ : coeficiente de determinação; $R_{a j}^{2}$ : coeficiente de determinação ajustado; syx: erro padrão da estimativa, em árv./ha; sy $x \%$ : erro padrão da estimativa em porcentagem; $D^{\prime}$ : valor tabelado do teste de KolmogorovSmirnov $\left(\alpha\right.$ : 0,01); $D_{\text {calc }}$ : valor calculado do teste de Kolmogorov-Smirnov; ${ }^{\text {ns: }}$ não significativo ao nível de $1 \%(\alpha: 0,01)$; $* *$ : significativo ao nível de $1 \%(\alpha: 0,01)$; I, II, III :classe de sítio I, classe de sítio II e classe de sítio III, as quais apresentam altura dominante aos 24 anos de idade de 35,91, 30,14 e 24,36 m, respectivamente.

Carelli Netto (2008) estudou a função Normal, Ln-Normal, Weibull e Gamma para representar a estrutura diamétrica de povoamentos de Pinus taeda de diferentes idades na região de Otacílio Costa (SC). Diferentemente deste trabalho, Carelli Netto (2008) concluiu que a função Normal e Ln-Normal apresentaram os melhores desempenhos para as idades iniciais e mais avançadas de Pinus taeda, respectivamente.

Machado et al. (2009b), Machado et al.(2010a) e Machado et al. (2010b), além das FDP testadas neste estudo, estudaram adicionalmente as funções Weibull 2P, Gamma (adaptada), de Péllico Netto, de Quadros e Exponencial para representar a distribuição de diferentes variáveis dendrométricas, para diversas espécies de um fragmento de Floresta Ombrófila Mista em Curitiba (PR). Diferentemente dos resultados encontrados neste estudo, a função de Weber apresentou a melhor aderência na maioria dos casos estudados por esses autores, principalmente para distribuições em forma de J-invertido e grau de assimetria à direita muito elevado.

Quanto aos resultados das estatísticas de ajuste e precisão, as funções com melhor aderência apresentaram altos valores de $R^{2}$ e $R_{a j}^{2}$. e baixos valores de $s y x$ e syx\%. Machado et al. (2009b), Machado et al. (2010a) e Machado et al. (2010b) encontram valores semelhantes para essas estatísticas de ajuste no estudo de funções de densidade probabilísticas para descrever a distribuição de diferentes variáveis de diversas espécies florestais no Brasil.

De acordo com as tabelas 4 e 5, nos ajustes das funções de densidade probabilísticas para as idades de 5 a 5,9 e de 6 a 6,9 anos, para a classe de sítio II, a função Normal e Sb de Johnson apresentaram os melhores desempenhos, com valores muito altos de $R^{2} \mathrm{e} R_{a j}^{2}$. e valores baixos para syx e 
syx\%, porém não apresentaram aderência satisfatória pelo teste de Kolmogorov-Smirnov. Segundo a tabela 1, para as classes de idade e de sítio citadas anteriormente, o número de observações é de 16.452 e 43.045, respectivamente. Já para as idades de 8 a 8,9 anos na classe de sítio III (Tabela 7), todas as funções apresentaram aderência satisfatória de acordo com o teste de Kolmogorov-Smirnov, mesmo aquelas que apresentaram valores mais baixos de $R^{2} \mathrm{e} R_{a j}^{2}$. e valores altos para $s y x$ e $s y x \%$. Ao observar a tabela 1, verifica-se que o número de observações desse último caso é de 351, muito abaixo dos valores observados para as idades de 5 a 5,9 anos e de 6 a 6,9 anos, para a classe de sítio II, citados anteriormente.

Tabela 6. Aderência das funções de densidade probabilísticas à distribuição diamétrica de povoamentos de Pinus taeda com idades de 7 a 7,9 anos, por classe de sítio.

Table 6. Adherence of the probability density functions to diameter distribution of Pinus taeda stands, with ages ranging from 7 to 7.9 years, by site classes.

\begin{tabular}{|c|c|c|c|c|c|c|c|c|}
\hline $\begin{array}{l}\text { Classe } \\
\text { de sítio }\end{array}$ & FDP & Ranking & $R^{2}$ & $R_{a j}^{2}$ & $\begin{array}{c}s y x \\
\text { (árv./ha) }\end{array}$ & syx $\%$ & $D^{\prime}$ & $D_{\text {calc }}$ \\
\hline \multirow{7}{*}{ I } & Sb de Johnson & $1^{\mathrm{a}}$ & 0,9992 & 0,9988 & 6,7 & 4,2 & \multirow{7}{*}{0,047} & $-0,009^{\mathrm{ns}}$ \\
\hline & Normal & $2^{\mathrm{a}}$ & 0,9991 & 0,9988 & 6,7 & 4,2 & & $-0,014^{\mathrm{ns}}$ \\
\hline & Weibull & $3^{\mathrm{a}}$ & 0,9981 & 0,9975 & 9,6 & 6,0 & & $-0,014^{\mathrm{ns}}$ \\
\hline & Beta & $4^{\mathrm{a}}$ & 0,9985 & 0,9974 & 9,9 & 6,2 & & $-0,022^{\mathrm{ns}}$ \\
\hline & Ln-Normal & $5^{\mathrm{a}}$ & 0,9843 & 0,9798 & 27,4 & 17,2 & & $-0,054 * *$ \\
\hline & Gamma & $6^{\mathrm{a}}$ & 0,9838 & 0,9758 & 30,0 & 18,9 & & $-0,066^{* *}$ \\
\hline & Weber & $7^{\mathrm{a}}$ & 0,9846 & 0,9723 & 32,1 & 20,2 & & $-0,058 * *$ \\
\hline \multirow{7}{*}{ II } & Sb de Johnson & $1^{\mathrm{a}}$ & 0,9996 & 0,9994 & 4,7 & 3,3 & \multirow{7}{*}{0,009} & $-0,008^{\mathrm{ns}}$ \\
\hline & Weibull & $2^{\mathrm{a}}$ & 0,9991 & 0,9989 & 6,7 & 4,7 & & $-0,010 * *$ \\
\hline & Normal & $3^{\mathrm{a}}$ & 0,9965 & 0,9957 & 13,2 & 9,3 & & $-0,027 * *$ \\
\hline & Beta & $4^{\mathrm{a}}$ & 0,9953 & 0,9922 & 17,8 & 12,5 & & $-0,035 * *$ \\
\hline & Gamma & $5^{\mathrm{a}}$ & 0,9814 & 0,9767 & 30,7 & 21,5 & & $-0,059 * *$ \\
\hline & Ln-Normal & $6^{\mathrm{a}}$ & 0,9770 & 0,9712 & 34,1 & 23,9 & & $-0,063 * *$ \\
\hline & Weber & $7^{\mathrm{a}}$ & 0,9737 & 0,9625 & 39,0 & 27,4 & & $-0,062 * *$ \\
\hline \multirow{7}{*}{ III } & Sb de Johnson & $1^{\mathrm{a}}$ & 0,9996 & 0,9994 & 5,9 & 3,1 & \multirow{7}{*}{0,028} & $-0,007^{\mathrm{ns}}$ \\
\hline & Weibull & $2^{\mathrm{a}}$ & 0,9992 & 0,9989 & 7,9 & 4,1 & & $-0,009^{\mathrm{ns}}$ \\
\hline & Beta & $3^{\mathrm{a}}$ & 0,9971 & 0,9932 & 19,5 & 10,2 & & $-0,027^{\mathrm{ns}}$ \\
\hline & Normal & $4^{\mathrm{a}}$ & 0,9942 & 0,9919 & 21,3 & 11,1 & & $-0,033^{*} *$ \\
\hline & Ln-Normal & $5^{\mathrm{a}}$ & 0,9721 & 0,9609 & 46,8 & 24,4 & & $-0,058 * *$ \\
\hline & Gamma & $6^{\mathrm{a}}$ & 0,9696 & 0,9575 & 48,8 & 25,4 & & $-0,068 * *$ \\
\hline & Weber & $7^{\mathrm{a}}$ & 0,9704 & 0,9483 & 53,8 & 28,0 & & $-0,079 * *$ \\
\hline
\end{tabular}

FDP: função de densidade probabilística; $R^{2}$ : coeficiente de determinação; $R_{a j}^{2}$ : coeficiente de determinação ajustado; syx: erro padrão da estimativa, em árv./ha; syx\%: erro padrão da estimativa em porcentagem; $D^{\prime}$ : valor tabelado do teste de KolmogorovSmirnov $(\alpha: 0,01) ; D_{\text {calc }}$ : valor calculado do teste de Kolmogorov-Smirnov; ${ }^{\mathrm{n}}$ : não significativo ao nível de $1 \%(\alpha: 0,01)$; $* *$ : significativo ao nível de $1 \%(\alpha: 0,01)$; I, II, III :classe de sítio I, classe de sítio II e classe de sítio III, as quais apresentam altura dominante aos 24 anos de idade de 35,91, 30,14 e 24,36 m, respectivamente.

Acredita-se que o grande número de observações utilizado para ajuste das funções influenciou o resultado final do teste de Kolmogorov-Smirnov, o qual indicou diferença estatística significativa $(\alpha=0,01)$ entre a frequências acumuladas observada e estimada pelas funções.

Téo et al. (2011b), no estudo da dinâmica da distribuição diamétrica para povoamentos de Pinus taeda L. na região de Caçador (SC), utilizaram apenas o teste de Kolmogorov-Smirnov para verificar a aderência das FDP. Esses autores relataram que o teste de Kolmogorov-Smirnov apresentou certa ineficiência para testar a aderência de funções aplicadas para descrever distribuições com grande número de observações, justamente o caso deste estudo. Esses mesmos autores reportaram que, quanto maior o número de observações da distribuição, menor é o valor de $D^{\prime}$ do teste, exigindo uma diferença muito pequena entre a frequência acumulada observada e a frequência acumulada esperada para indicar boa aderência da função. 
Tabela 7. Aderência das funções de densidade probabilísticas à distribuição diamétrica de povoamentos de Pinus taeda com idades de 8 a 8,9 anos, por classe de sítio.

Table 7. Adherence of the probability density functions to diameter distribution of Pinus taeda stands, with ages ranging from 8 to 8.9 years, by site classes.

\begin{tabular}{|c|c|c|c|c|c|c|c|c|}
\hline $\begin{array}{l}\text { Classe } \\
\text { de sítio }\end{array}$ & FDP & Ranking & $R^{2}$ & $R_{a j}^{2}$ & $\begin{array}{c}\operatorname{syx} \\
\text { (árv./ha) }\end{array}$ & $\boldsymbol{s y} \boldsymbol{x} \%$ & $D^{\prime}$ & $D_{c a l c}$ \\
\hline \multirow{7}{*}{ I } & Weibull & $1^{\mathrm{a}}$ & 0,9909 & 0,9886 & 17,7 & 12,0 & \multirow{7}{*}{0,045} & $-0,010^{\mathrm{ns}}$ \\
\hline & Sb de Johnson & $2^{\mathrm{a}}$ & 0,9903 & 0,9839 & 20,3 & 13,7 & & $-0,009^{\mathrm{ns}}$ \\
\hline & Normal & $3^{\mathrm{a}}$ & 0,9828 & 0,9785 & 24,4 & 16,4 & & $-0,036^{\mathrm{ns}}$ \\
\hline & Beta & $4^{\mathrm{a}}$ & 0,9866 & 0,9777 & 24,8 & 16,7 & & $-0,038^{\mathrm{ns}}$ \\
\hline & Ln-Normal & $5^{\mathrm{a}}$ & 0,9505 & 0,9381 & 41,3 & 27,9 & & $-0,079 * *$ \\
\hline & Gamma & $6^{\mathrm{a}}$ & 0,9503 & 0,9379 & 41,4 & 27,9 & & $-0,096 * *$ \\
\hline & Weber & $7^{\mathrm{a}}$ & 0,9525 & 0,9321 & 43,3 & 29,2 & & $-0,082^{* *}$ \\
\hline \multirow{7}{*}{ II } & Weibull & $1^{\mathrm{a}}$ & 0,9982 & 0,9977 & 9,1 & 5,6 & \multirow{7}{*}{0,013} & $-0,008^{\mathrm{ns}}$ \\
\hline & Sb de Johnson & $2^{\mathrm{a}}$ & 0,9985 & 0,9977 & 9,2 & 5,6 & & $-0,008^{\mathrm{ns}}$ \\
\hline & Beta & $3^{\mathrm{a}}$ & 0,9937 & 0,9887 & 20,3 & 12,5 & & $-0,036 * *$ \\
\hline & Normal & $4^{\mathrm{a}}$ & 0,9880 & 0,9846 & 23,8 & 14,6 & & $-0,044 * *$ \\
\hline & Gamma & $5^{\mathrm{a}}$ & 0,9639 & 0,9536 & 41,2 & 25,3 & & $-0,087 * *$ \\
\hline & Ln-Normal & $6^{\mathrm{a}}$ & 0,9570 & 0,9447 & 45,0 & 27,6 & & $-0,089 * *$ \\
\hline & Weber & $7^{\mathrm{a}}$ & 0,9575 & 0,9363 & 48,3 & 29,7 & & $-0,077 * *$ \\
\hline \multirow{7}{*}{ III } & Weibull & $1^{\mathrm{a}}$ & 0,9968 & 0,9953 & 17,0 & 8,2 & \multirow{7}{*}{0,087} & $0,021^{\mathrm{ns}}$ \\
\hline & Sb de Johnson & $2^{\mathrm{a}}$ & 0,9963 & 0,9925 & 21,3 & 10,3 & & $0,025^{\mathrm{ns}}$ \\
\hline & Normal & $3^{\mathrm{a}}$ & 0,9923 & 0,9885 & 26,5 & 12,8 & & $-0,045^{\mathrm{ns}}$ \\
\hline & Ln-Normal & $4^{\mathrm{a}}$ & 0,9809 & 0,9713 & 41,9 & 20,2 & & $-0,069^{\mathrm{ns}}$ \\
\hline & Beta & $5^{\mathrm{a}}$ & 0,9900 & 0,9699 & 42,9 & 20,7 & & $-0,056^{\mathrm{ns}}$ \\
\hline & Gamma & $6^{\mathrm{a}}$ & 0,9802 & 0,9703 & 42,6 & 20,6 & & $-0,071^{\mathrm{ns}}$ \\
\hline & Weber & $7^{\mathrm{a}}$ & 0,9769 & 0,9306 & 65,1 & 31,4 & & $-0,063^{\mathrm{ns}}$ \\
\hline
\end{tabular}

FDP: função de densidade probabilística; $R^{2}$ : coeficiente de determinação; $R_{a j}^{2}$ : coeficiente de determinação ajustado; syx: erro padrão da estimativa, em árv./ha; syx\%: erro padrão da estimativa em porcentagem; $D^{\prime}$ : valor tabelado do teste de KolmogorovSmirnov $(\alpha: 0,01) ; D_{\text {calc }}$ : valor calculado do teste de Kolmogorov-Smirnov; ${ }^{\text {ns: }}$ não significativo ao nível de $1 \%(\alpha: 0,01)$; $* *$ : significativo ao nível de $1 \%(\alpha: 0,01)$; I, II, III :classe de sítio I, classe de sítio II e classe de sítio III, as quais apresentam altura dominante aos 24 anos de idade de 35,91, 30,14 e 24,36 m, respectivamente.

Essa sensibilidade apresentada pelo teste de Kolmogorov-Smirnov demonstra a necessidade de utilização de mais critérios para avaliação do desempenho das funções de densidade probabilísticas na representação da distribuição diamétrica.

Asfiguras1 e 2apresentam as distribuições diamétricas observada e estimada pela função de densidade probabilística de melhor desempenho para povoamentos de Pinus taeda na região de Caçador (SC), para as classes de idade de 5 a 5,9 anos, 6 a 6,9 anos,7 a 7,9 anos e 8 a 8,9 anos e classes de sítio I, II e III.

Os povoamentos de Pinus taeda da região de Caçador (SC) apresentaram distribuição diamétrica unimodal em todas as classes de idade e classes de sítio avaliadas neste estudo, como pode ser verificado nas figuras 1 e 2 .

Quanto ao efeito das diferentes classes de idade sobre a distribuição diamétrica, pode-se observar que o diâmetro máximo, representado pela cauda direita da curva de distribuição, aumenta de acordo com a idade para as classes de sítio I e II, entretanto isso não ocorre para a classe de sítio III. Comparando-se a distribuição diamétrica das classes de idade de 7 a 7,9 anos e de 8 a 8,9 anos para a classe de sítio III, há uma redução do diâmetro máximo de $25,5 \mathrm{~cm}$ para $22,5 \mathrm{~cm}$.

Quanto às classes diamétricas com maiores frequências, percebe-se que, à medida que a idade avança, os valores da moda se deslocam para a direita, fato observado nas curvas de distribuição das classes de sítio I e II. Esse deslocamento das curvas de distribuição dos diâmetros para a direita com o avanço da idade também foi encontrado por Carelli Netto (2008), Leite et al. (2005), Machado et al.(2006), Nogueira et al. (2006) e Téo et al.(2011b), estudando a dinâmica da distribuição diamétrica de povoamentos de P. taeda, Eucalyptu ssp., Mimosa scabrella Bentham, Tectona grandis L. F., e P. taeda, respectivamente, em diversas regiões do Brasil. 

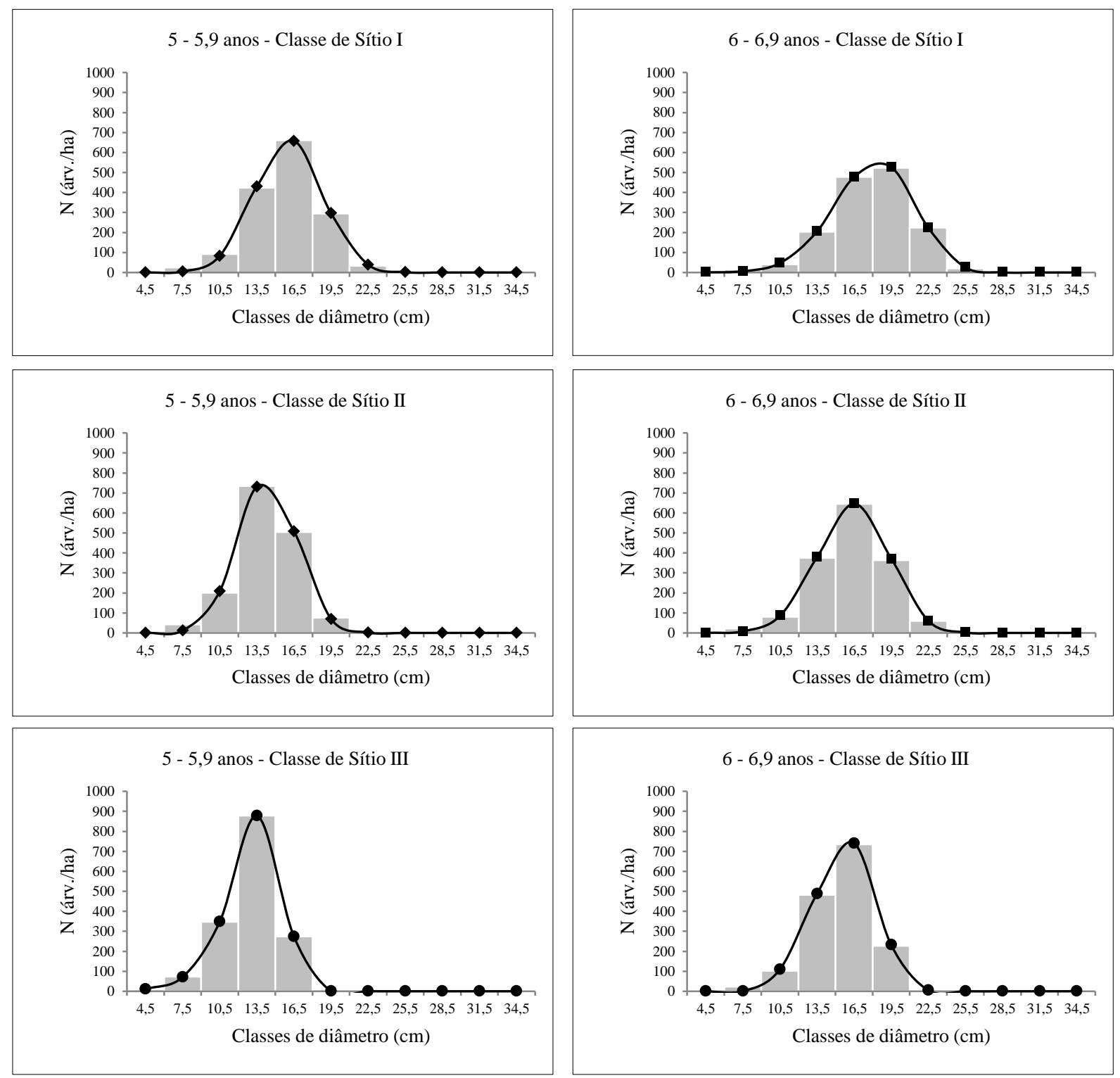

Frequência observada; - Weibull;

- Sb de Johnson.

Classe de sítio I = Índice de sítio $24=35,91 \mathrm{~m}$; Classe de sítio II = Índice de sítio $\mathrm{O}_{24}=30,14 \mathrm{~m}$; Classe de sítio III = Índice de sítio $24=24,36 \mathrm{~m}$.

Figura 1. Distribuições diamétricas observada e estimada pela função de densidade probabilística de melhor desempenho para povoamentos de Pinus taeda para as idades de 5 a 6,9 anos.

Figure 1. Observed diameter distribution and estimated diameter distribution by the best performance probability density function for Pinus taeda stands for ages from 5 to 6.9 years.

Ao comparar a diferença da distribuição diamétrica de acordo com cada classe de sítio, percebese um achatamento nas curvas em função da melhora da classe de sítio. A curva de distribuição diamétrica da classe de sítio III possui maior frequência em poucas classes de diâmetro; já a curva que representa a classe de sítio II possui uma maior amplitude dos diâmetros, quando comparada com a classe de sítio III. O mesmo comportamento pode ser observado para a distribuição diamétrica da classe de sítio I com relação à classe de sítio II. 

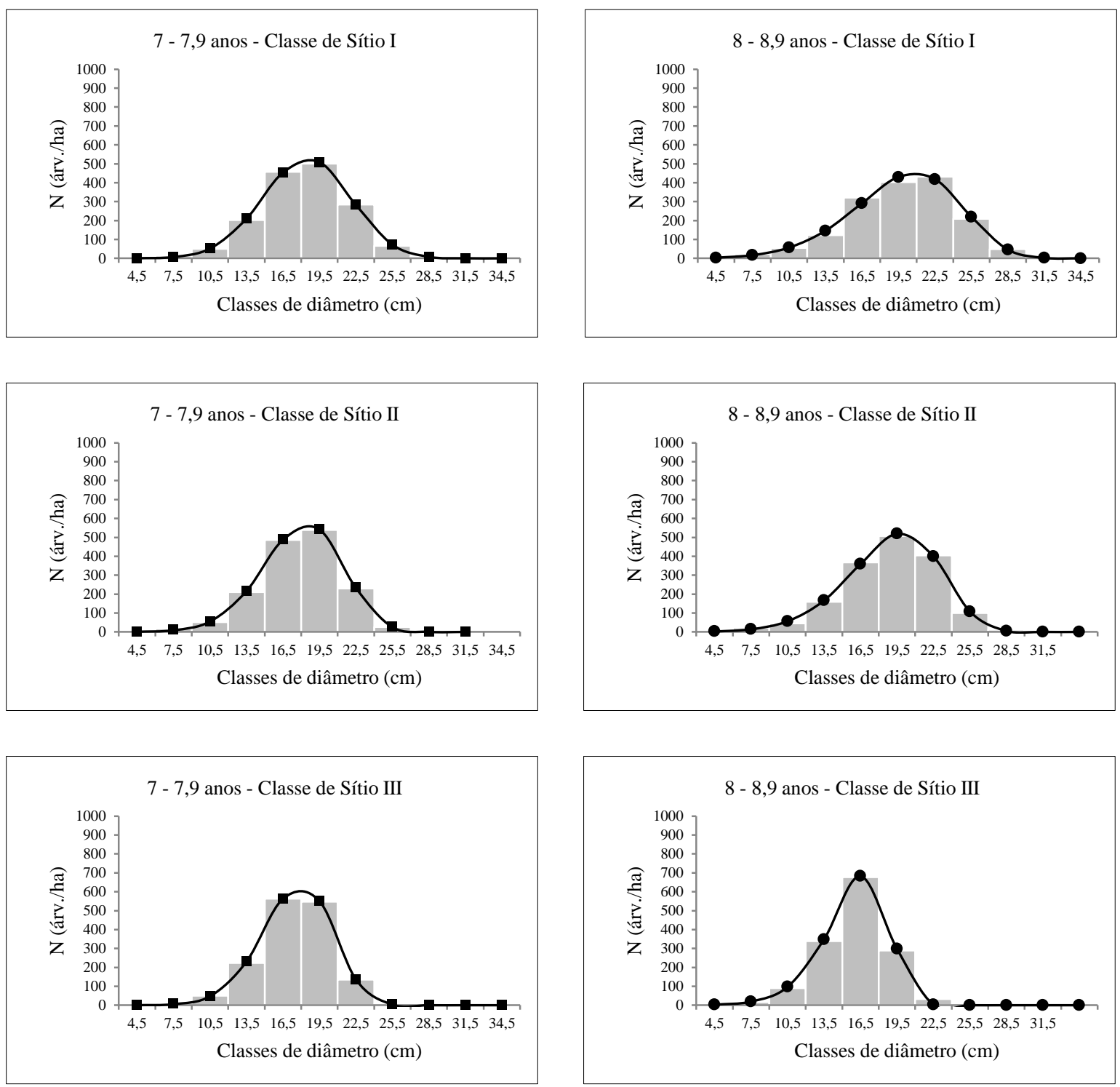

Frequência observada;

Weibull;

Sb de Johnson.

Classe de sítio I = Índice de sítio $_{24}=35,91 \mathrm{~m}$; Classe de sítio II = Índice de sítio ${ }_{24}=30,14 \mathrm{~m}$; Classe de sítio III = Índice de sítio $24=$ $24,36 \mathrm{~m}$.

Figura 2. Distribuição diamétrica observada e estimada pela função de densidade probabilística de melhor desempenho para povoamentos de Pinus taeda para idades 7 a 8,9 anos.

Figure 2. Observed diameter distribution and estimated diameter distribution by the best performance probability density function for Pinus taeda stands for ages form 7 to 8.9 years.

Ao comparar a distribuição diamétrica da menor para a maior idade, e da classe de sítio III para a classe de sítio I, verificou-se que ocorreu o aumento na amplitude total dos diâmetros. Esse aumento da amplitude dos diâmetros com o passar dos anos também foi constatado por Carelli Netto (2008), Leite et al.(2005), Machado et al. (2006), Nogueira et al. (2006), Machado et al. (2008) e Téo et al.(2011b), trabalhando com diferentes espécies em diferentes regiões. 
Machado et al. (2008) estudaram o efeito do sítio e da densidade inicial na distribuição diamétrica de Mimosa scabrella na Região Metropolitana de Curitiba, com idades de 4 a 7,5 anos. Da mesma forma que ocorreu neste estudo, evidenciou-se que as curvas de distribuição diamétrica se deslocaram para a direita nos sítios mais produtivos e quanto menores as densidades iniciais dos povoamentos.

A tabela 8 apresenta os parâmetros de assimetria e curtose da distribuição diamétrica dos povoamentos de Pinus taeda da região de Caçador (SC), para as diferentes classes de idade e de sítio florestal.

Tabela 8. Assimetria e curtose da distribuição diamétrica de povoamentos de Pinus taeda para diferentes classes de idade e de sítio.

Table 8. Skewness and kurtosis of the diameter distribution for Pinus taeda stands for different age and site classes.

\begin{tabular}{lccc}
\hline Classes de idade(anos) & Classes de sítio & Assimetria $\left(\gamma_{\mathbf{1}}\right)$ & Curtose $\left(\boldsymbol{\gamma}_{\mathbf{2}}\right)$ \\
\hline \multirow{3}{*}{$5-5,9$} & I & $-0,50773$ & 1,03084 \\
& II & $-0,48225$ & 0,84360 \\
& III & $-0,54678$ & 0,84229 \\
\hline \multirow{3}{*}{6,9} & I & $-0,52836$ & 0,84119 \\
& II & $-0,44055$ & 0,66241 \\
\hline \multirow{3}{*}{$-7,9$} & III & $-0,54125$ & 0,63971 \\
\hline \multirow{3}{*}{$8-8,9$} & I & $-0,26040$ & 0,83416 \\
& II & $-0,47335$ & 0,81574 \\
& III & $-0,51171$ & 0,61378 \\
\hline III & I & $-0,45256$ & 0,56067 \\
& II & $-0,56693$ & 0,79426 \\
& III & $-0,66482$ & 1,12847 \\
\hline
\end{tabular}

I, II, III: classe de sítio I, classe de sítio II e classe de sítio III, as quais apresentam altura dominante aos 24 anos de idade de 35,91, 30,14 e 24,36 m, respectivamente.

Em todas as classes de idade e classes de sítio, a assimetria da distribuição diamétrica foi negativa ou à direita $\left(\gamma_{1}<0\right)$. Já a curtose foi positiva $\left(\gamma_{2}>0\right)$, também chamada de leptocúrtica, ou seja, o topo da curva de distribuição é mais pontiagudo comparando-se à distribuição normal, como pode ser observado na tabela 8 .

Em acordo com os resultados encontrados neste estudo, Téo et al. (2011b) encontraram assimetria negativa ou à direita e curtose positiva para distribuição diamétrica de Pinus taeda na região de Caçador (SC), para as idades de 5 a 7,9 anos e de 9 a 11,9 anos. No entanto, Carelli Netto (2008) encontrou assimetria positiva para povoamentos dessa espécie com idades de 6 a 18 anos.

De acordo com os resultados da tabela 8 , não há uma tendência clara de aumento ou diminuição dos parâmetros de assimetria e curtose com relação às diferentes classes de idade. No entanto, há uma tendência de que no sítio menos produtivo (classe de sítio III) a distribuição diamétrica é mais assimétrica negativa. Essa tendência é mais evidente para as classes de idade de 7 a 7,9 e 8 a 8,9 anos.

Quanto à curtose, para as classes de idade de 5 a 7,9 anos há uma diminuição dos valores do sítio mais produtivo para o sítio menos produtivo, entretanto, para a classe de idade de 8 a 8,9 anos, o inverso pode ser observado. No entanto, de acordo com Marques (2003), somente faz sentido calcular o coeficiente de curtose para distribuições simétricas ou aproximadamente simétricas, o que não é o caso das distribuições analisadas neste estudo.

Machado et al. (2008), baseados em observações das curvas de distribuição diamétrica de Mimosa scabrella, observaram que, à medida que a produtividade do sítio reduz, o desenvolvimento das árvores em diâmetro é menor, o que implica uma frequência observada com assimetria menos acentuada, inverso da tendência encontrada neste estudo.

Finger (1982), estudando a distribuição de diâmetros em Acacia mearnsii De Wild em diferentes idades, constatou que, para as idades de 4,5, 5,5, 6,5 e 7,5 anos, a distribuição dos diâmetros foi assimétrica negativa e a curtose foi platicúrtica. 
Machado et al. (2009a) e Machado et al. (2010a), estudando a distribuição de diâmetros, altura total, área transversal e volume individual de Araucaria angustifolia em Curitiba (PR), encontraram que a distribuição diamétrica foi perfeitamente simétrica e platicúrtica. A distribuição das alturas das árvores apresentou-se muito próxima a uma distribuição normal, levando em consideração tanto assimetria como curtose. Já as distribuições da área transversal e do volume apresentaram assimetria positiva.

De acordo com Machado et al. (2009b), a distribuição diamétrica de Cedrela fissilis Vell. E Gochnati apolymorpha (Less.) Cabr. apresentaram-se com assimetria positiva em um fragmento de Floresta Ombrófila Mista em Curitiba (PR).

\section{CONCLUSÕES}

De acordo com os resultados da presente pesquisa, foi possível concluir que:

- As funções $\mathrm{Sb}$ de Johnson e Weibull 3P apresentaram os melhores resultados na representação da dinâmica da distribuição diamétrica de povoamentos de Pinus taeda na região de Caçador (SC), enquanto as funções Gamma, Ln-Normal e Weber obtiveram os piores resultados.

- Quanto à avaliação da aderência das funções de densidade probabilísticas, recomenda-se a utilização das estatísticas $R^{2}, R_{a j}^{2}$. syx e $s y x \%$, além do teste de Kolmogorov-Smirnov em todos os casos, sobretudo quando o número de observações for maior do que 5.000.

- De maneira geral, há um aumento da amplitude dos valores dos diâmetros e um achatamento da distribuição diamétrica com o avanço da idade e com a melhora da produtividade do sítio. Ainda com relação ao avanço da idade, há um aumento dos valores do diâmetro máximo e do diâmetro modal das distribuições para as classes de sítio de maior produtividade, porém o mesmo não ocorre para a pior classe de sítio (classe de sítio III).

- Em todas as classes de idade e classes de sítio dos povoamentos de Pinus taeda, a assimetria da distribuição diamétrica foi negativa ou à direita $\left(\gamma_{1}<0\right)$ e a curtose foi positiva $\left(\gamma_{2}>0\right)$, ou leptocúrtica.

\section{REFERÊNCIAS}

ARCE, J. E. Modelagem da estrutura de florestas clonais de Populus deltoides Marsh. através de distribuições diamétricas probabilísticas. Ciência Florestal, Santa Maria, v. 14, n. 1, p. 149 - 164, 2004.

BARTOSZECK, A. C. de P. e S.; MACHADO, S. do A.; FIGUEIREDO FILHO, A.; OLIVEIRA, E. B. A distribuição diamétrica para bracatingais em diferentes idades, sítios e densidades na Região Metropolitana de Curitiba. Floresta, Curitiba, v. 34, n. 3, p. 305 - 323, 2004.

CARELLI NETTO, C. Dinâmica da distribuição diamétrica de povoamentos de Pinus taeda $\mathbf{L}$. em diferentes idades e espaçamentos. 105 f. Dissertação (Mestrado em Engenharia Florestal) Universidade Federal de Santa Maria, Santa Maria, 2008.

ClUTtER, J. L.; FORTSON, J. C.; PIENNAR, L. V.; BRISTER, G. H.; BAILEY, R. L. Timber management: a quantitative approach. New York: John Wiley \& Sons, 1983. 333 p.

DRAPER, N. R.; SMITH, H. Applied regression analysis. 3 ed. New York: John Wiley \& Sons, 1998. $706 \mathrm{p}$.

FIGURA, M. A. A distribuição de Weibull na descrição da estrutura diamétrica de Eucalyptus grandis: Um enfoque sobre o método dos momentos. 98 f. Dissertação (Mestrado em Engenharia Florestal) - Universidade Federal do Paraná, Curitiba, 2010.

FINGER, C. A. G. Distribuição de diâmetros em Acácia Negra (Acacia mearnsii de Wild), em diferentes povoamentos e idades. 146 f. Dissertação (Mestrado em Ciências Florestais) - Universidade Federal do Paraná, Curitiba, 1982.

LEITE, H. G.; NOGUEIRA, G. S.; CAMPOS, J. C. C.; SOUZA, A. L.; CARVALHO, A. Avaliação de um modelo de distribuição diamétrica ajustado para povoamento de Eucalyptus sp. submetidos a desbaste. Revista Árvore, Viçosa, v. 29, n. 2, p. 271-280, 2005. 
LOETSCH, F.; ZÖHRER, F.; HALLER, K. E. Forest Inventory. München: BLV Verlagsgesellschaft, 1973. v. 2. 469 p.

MACHADO, S. do A.; AUGUSTYNCZIK, A. L. D.; NASCIMENTO, R. G. M. do; FIGURA, M. A.; SILVA, L. C. R. da; MIGUEL, E. P.; TÉO, S. J. Distribuição diamétrica de Araucaria angustifolia (Bert.) O. Ktze. em um fragmento de Floresta Ombrófila Mista. Scientia Agraria, Curitiba, v. 10, n. 2, p. 103 110, 2009a.

MACHADO, S. do A.; AUGUSTYNCZIK, A. L. D.; NASCIMENTO, R. G. M. do; TÉO, S. J.; MIGUEL, E. P.; FIGURA, M. A.; SILVA, L. C. R. da. Funções de distribuição diamétrica em um fragmento de Floresta Ombrófila Mista. Ciência Rural, Santa Maria, v. 39, n. 8, p. 2428 - 2434. 2009b.

MACHADO, S. do A.; BARTOSZECK, A. C. de P. e S.; FIGUEIREDO FILHO, A.; OLIVEIRA, E. B. de. Dinâmica da distribuição diamétrica de bracatingais na Região Metropolitana de Curitiba. Revista Árvore, Viçosa, v. 30, n. 5, p. 759 - 768, 2006.

MACHADO, S. do A.; BARTOSZECK, A. C. de P. e S.; FIGUEIREDO FILHO, A.; OLIVEIRA, E. B. de. Efeito da densidade e do sítio sobre as curvas de distribuição diamétrica em bracatingais nativos (Mimosa scabrella Benth.) da Região Metropolitana de Curitiba. Ambiência, Guarapuava, v. 4, n. 1, p. 37 - 50, 2008.

MACHADO, S. do A.; NASCIMENTO, R. G. M. do; MIGUEL, E. P.; TÉO, S. J.; AUGUSTYNCZIK, A. L. D. Distribution of total height, transverse area and individual volume for Araucaria angustifólia (Bert.) O. Kuntze. Cerne, Lavras, v. 16, n. 1, p. 12 - 21, 2010a.

MACHADO, S. do A.; SANTOS, A. A. P. dos; NASCIMENTO, R. G. M. do; AUGUSTYNCZIK, A. L. D.; ZAMIN, N. T. Modelagem da distribuição diamétrica de quatro espécies de Lauraceae em um fragmento de Floresta Ombrófila Mista. Revista Ciências Exatas e Naturais, Guarapuava, v. 12, n. 1, p. $91-105,2010$ b.

MARQUES, J. M. Estatística: cursos de engenharia. Curitiba: UFPR, 2003. 191 p. Apostila.

MONTGOMERY, D. C.; PECK, E. A.; VINING, G. G. Introduction to linear regression analysis.4 ed. New York: John Wiley \& Sons, 2006. 612 p.

NOGUEIRA, G. S.; LEITE, H. G.; CAMPOS, J. C. C.; TAKIZAWA, F. H.; COUTO, L. Avaliação de um modelo de distribuição diamétrica ajustado para povoamentos de Tectona grandis submetidos a desbaste. Revista Árvore, Viçosa, v. 30, n. 3, p. 377 - 387, 2006.

PRATES, A. M. M.; MANZOLLI, J. I.; MIRA, M. A. F. B. Geografia física de Santa Catarina. Florianópolis: Editora Lunardelli, 1989. 112 p.

PRODAN, M.; PETERS, R.; COX, F.; REAL, P. Mensura Forestal. San José: Instituto Interamericano de Cooperación para la Agricultura (IICA), 1997. 586 p.

TÉO, S. J.; BRESSAN, D. R.; COSTA, R. H. da. Uso de modelos estatísticos para classificação de sítios em povoamentos de Pinus taeda na região de Caçador, SC. Floresta, Curitiba, v. 41, n. 1, p. 179 - 188, 2011a.

TÉO, S. J.; ROCHA, S. P. da; BORTONCELlO, A. C.; PAZ, R. A.; COSTA, R. H. da. Dinâmica da distribuição diamétrica de povoamentos de Pinus taeda na região de Caçador, SC. Pesquisa Florestal Brasileira, Colombo, v. 31, n. 67, p. 183 - 192, 2011 b. 